PHYSICAL REVIEW B 90, 140503(R) (2014)

S

\title{
Experimental observation of the breaking and recombination of single Cooper pairs
}

\author{
N. J. Lambert, M. Edwards, and A. A. Esmail \\ Microelectronics Group, Cavendish Laboratory, University of Cambridge, Cambridge CB3 OHE, United Kingdom
}

F. A. Pollock

Atomic \& Laser Physics, Clarendon Laboratory, University of Oxford, Oxford OX1 3PU, United Kingdom

S. D. Barrett

Controlled Quantum Dynamics Theory, Imperial College London, South Kensington, London SW7 2AZ, United Kingdom

B. W. Lovett

SUPA, School of Physics and Astronomy, University of St. Andrews, St. Andrews KY16 9SS, United Kingdom and Department of Materials, Oxford University, Oxford OX1 3PH, United Kingdom

A. J. Ferguson*

Microelectronics Group, Cavendish Laboratory, University of Cambridge, Cambridge CB3 OHE, United Kingdom

(Received 28 March 2014; revised manuscript received 20 July 2014; published 9 October 2014)

\begin{abstract}
We observe the real-time breaking of single Cooper pairs by monitoring the radio-frequency impedance of a superconducting double quantum dot. The Cooper pair breaking rate in the microscale islands of our device decreases as temperature is reduced, saturating at $2 \mathrm{kHz}$ for temperatures beneath $100 \mathrm{mK}$. In addition, we measure in real time the quasiparticle recombination into Cooper pairs. Analysis of the recombination rates shows that, in contrast to bulk films, a multistage recombination pathway is followed.
\end{abstract}

DOI: 10.1103/PhysRevB.90.140503

Unpaired electronic excitations-quasiparticles-play an important role in determining the behavior of superconducting electrical devices. They lead to even-odd parity effects in Coulomb blockade nanostructures [1,2]; they act as a source of decoherence in superconducting qubits [3]; they cause generation-recombination noise in superconducting resonators [4]; they may be important in superconductingnormal devices for Majorana fermionics [5]; and, importantly, they enable the detection of far-infrared light, for example, in kinetic inductance detectors [6]. In this Rapid Communication we investigate the generation and recombination of single quasiparticle pairs in a superconducting double dot (SDD), a Coulomb blockade nanostructure. Double quantum dots have been widely investigated in the context of semiconducting spin qubits where they enable electrostatic control and measurement over electron spins and spin pairs [7]. Previously, semiconductor double dots have been integrated with superconducting leads, allowing electrostatically tunable supercurrents [8] and the splitting of Cooper pair currents into spatially separated and correlated electron currents [9-11]. However, apart from an early study investigating the superconducting double quantum dot as a qubit architecture [12], there have been few studies of this system, thus motivating our current work.

We investigate the quasiparticle dynamics in the SDD, therefore, our results are relevant to the long-standing quasiparticle poisoning problem in superconducting qubits $[13,14]$. It has long been known that incoherent quasiparticle tunneling interrupts the coherent tunneling of Cooper pairs. Quasiparticle poisoning is hence a serious issue in charge-based

\footnotetext{
*ajf1006@cam.ac.uk
}

PACS number(s): 74.50.+r, 85.25.Oj, 74.45.+c, 85.25.Cp

superconducting qubits [15] and has recently been shown to be relevant in the case of low-charging energy transmon qubits [16]. Experiments on superconducting qubits have shown that by taking extreme care over filtering infrared radiation it is possible to extend coherence times, presumably because of the lower quasiparticle temperatures achieved [17]. Quasiparticle tunneling into a Cooper pair box has been used to detect far-infrared radiation from a blackbody source with a noise-equivalent power of less than $10^{-19} \mathrm{~W} / \mathrm{Hz}^{1 / 2}$, potentially providing a successor technology to kinetic inductance detectors [18]. In parallel, studies on superconducting resonators have shown a saturation of the quasiparticle population at a relatively high temperature of $140 \mathrm{mK}$ [19]. It remains an experimental challenge to reduce the quasiparticle temperature towards the base lattice temperature in a dilution refrigerator.

Our SDD consists of two superconducting Al islands connected by an $R=7 \mathrm{k} \Omega$ tunnel barrier. Each island is also connected to normal metal $\left(\mathrm{Al}_{0.98} \mathrm{Mn}_{0.02}\right)$ source and drain reservoirs by relatively opaque $(R=4 \mathrm{M} \Omega)$ tunnel barriers [Fig. 1(a)]. A pair of electrostatic gates addresses each island, allowing control over the charge state. The SDD pattern is defined by electron beam lithography, the metal deposited by multiple-angle thermal evaporation and the tunnel barriers formed by controlled in situ oxidation. In previous work we characterized the normal state behavior of similar devices [20], but here we focus on properties arising from superconductivity.

We measure the amplitude and phase of a low power $(-121 \mathrm{dBm})$ radio-frequency signal reflected from a lumped element resonant circuit which, along with the SDD, is maintained at the base temperature of a dilution refrigerator [Fig. 1(b)]. Throughout the work reported here, our main observable is a phase shift associated with the electric polarizability of the Cooper pair and quasiparticle states. 
(a)

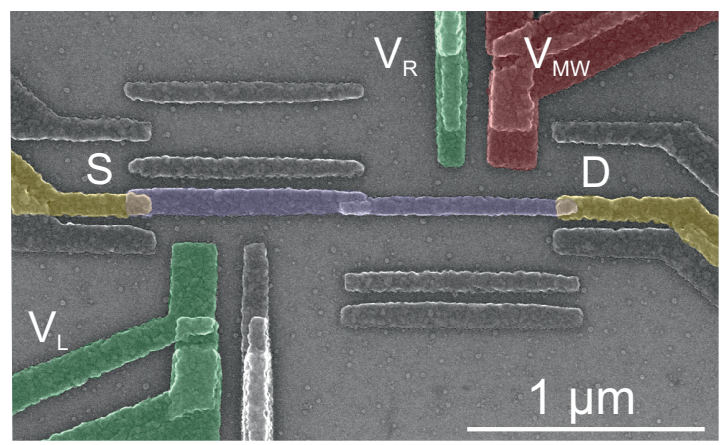

(b)

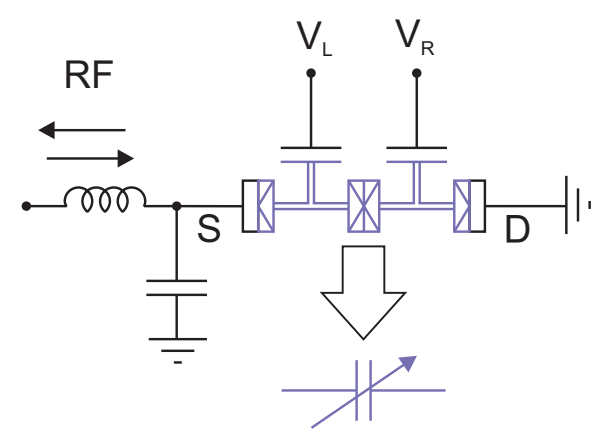

FIG. 1. (Color online) Device and experimental configuration. (a) Scanning electron micrograph of a superconducting double dot device. The false color regions show the dc gates (green), the microwave gate (red), the source and drain contacts (yellow), and the islands (purple). Uncolored metal regions are artifacts of the triple angle evaporation process. (b) The device is embedded in a lumped element $L C$ resonant circuit, with $L=510 \mathrm{nH}$ and $C=0.41 \mathrm{pF}$. The $\mathrm{V}$-shaped marks denote superconducting electrodes. The circuit resonates at $349 \mathrm{MHz}$ with a loaded $Q$ factor of $\approx 50$.

In the case of the Cooper pair states, this polarizability is usually referred to as the "quantum capacitance" [21,22] and is comparable in magnitude to the junction capacitances of the device $\left(C_{Q} \sim \mathrm{fF}\right)$. The quantum capacitance is given by $C_{Q}=C_{\text {geom }}-d^{2} E / d V^{2}$, where $V$ is the voltage on the source contact and $E$ is the energy of the state probed. Therefore the biggest changes are observed near an anticrossing and no quantum capacitance is observed in classical Coulomb blockade devices. The quantum capacitance has been useful in measurements of quasiparticle tunneling in Cooper pair boxes [23], and it has also been used for spin readout in the case of a semiconductor double quantum dot [24].

At millikelvin temperatures the quantized nature of charge becomes apparent. When the gate potentials are changed, a honeycomb pattern characteristic of Coulomb blockade in double quantum dots is observed [25-27]. Since the dot-lead tunnel barriers are relatively opaque, the resistance of the sample is poorly matched by the tank circuit and we see, in the averaged reflected radio-frequency signal, a purely dispersive contribution as Cooper pairs are transferred between the dots [Fig. 2(a)]. Meanwhile there is no dispersive or dissipative signal due to the Andreev-reflection processes which changes the total charge on the SDD, presumably due to the low rates of these processes [28]. Using the island $\left(E_{C L}=314 \mu \mathrm{eV}\right.$,

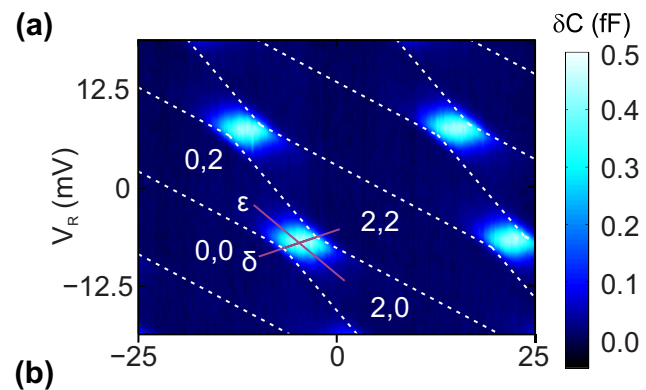

(b)

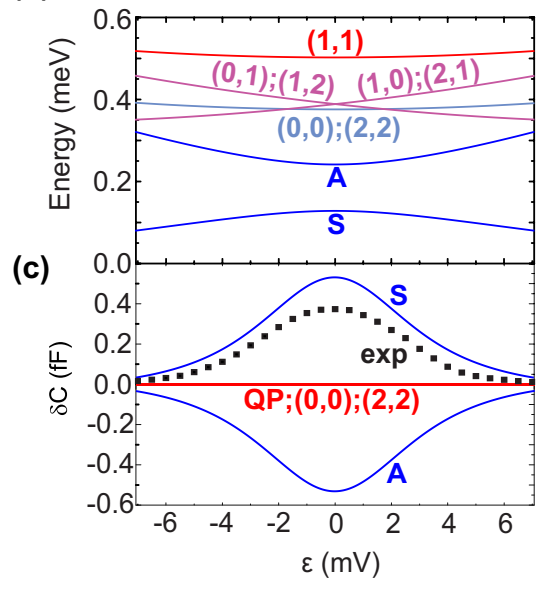

FIG. 2. (Color online) Quantum capacitance. (a) Averaged measurement of capacitance as a function of the dc control gates. The charge states are shown and a reference state $(0,0)$ is arbitrarily chosen. We illustrate the detuning axes $\delta$ and $\epsilon$. (b) The calculated device band structure as a function of $\epsilon$ detuning for different island charge states. The calculation was performed with experimentally determined values for the device energy parameters. (c) A comparison of measured and calculated values of $\delta C$ for the symmetric (ground state, labeled $S$ ) and the antisymmetric (labeled $A$ ) Cooper pair bands and the quasiparticle states.

$\left.E_{C R}=227 \mu \mathrm{eV}\right)$ and interdot $\left(E_{C M}=88 \mu \mathrm{eV}\right)$ charging energies, and the Josephson energy (measured by microwave spectroscopy to be $110 \pm 10 \mu \mathrm{eV}$ ), which couples the Cooper pair charge states, we determine the Helmholtz free energies of the charge states. We plot in Fig. 2(b) the expected cross section of the energy states for a detuning axis labeled $\epsilon$ whose modification causes charge transfer between the dots. The energetics are similar to those of a semiconductor double dot [25], except for the quasiparticle charge states which have an additional free energy cost of $\tilde{\Delta}$ per quasiparticle excitation. The Helmholtz free energy is given by $F=U-T S$, where $U$ is the internal energy, $T$ is the temperature, and $S$ the entropy, and so $\tilde{\Delta}=\Delta-k_{b} T \ln N_{\text {eff }}$, where $\Delta$ is the superconducting gap and $N_{\text {eff }}=2 \sqrt{2} V \rho(0) \sqrt{\Delta k_{B} T} \sim 5000$ is the effective number of states available for occupation by an excited quasiparticle [1]. Here, $\rho(0)$ is the single-spin density of states at the Fermi surface for normal state aluminum and $V \approx 4 \times 10^{3} \mu \mathrm{m}^{3}$ is the island volume.

We calculate $C_{Q}(\epsilon)$ for the various charge states and find good agreement between the measured signal and the expected value for the ground state [Fig. 2(c)]. The experimental data underestimate the magnitude of $C_{Q}$ due to the finite occupancy of excited states with $C_{Q}<0$, or $C_{Q}=0$. 
(a)
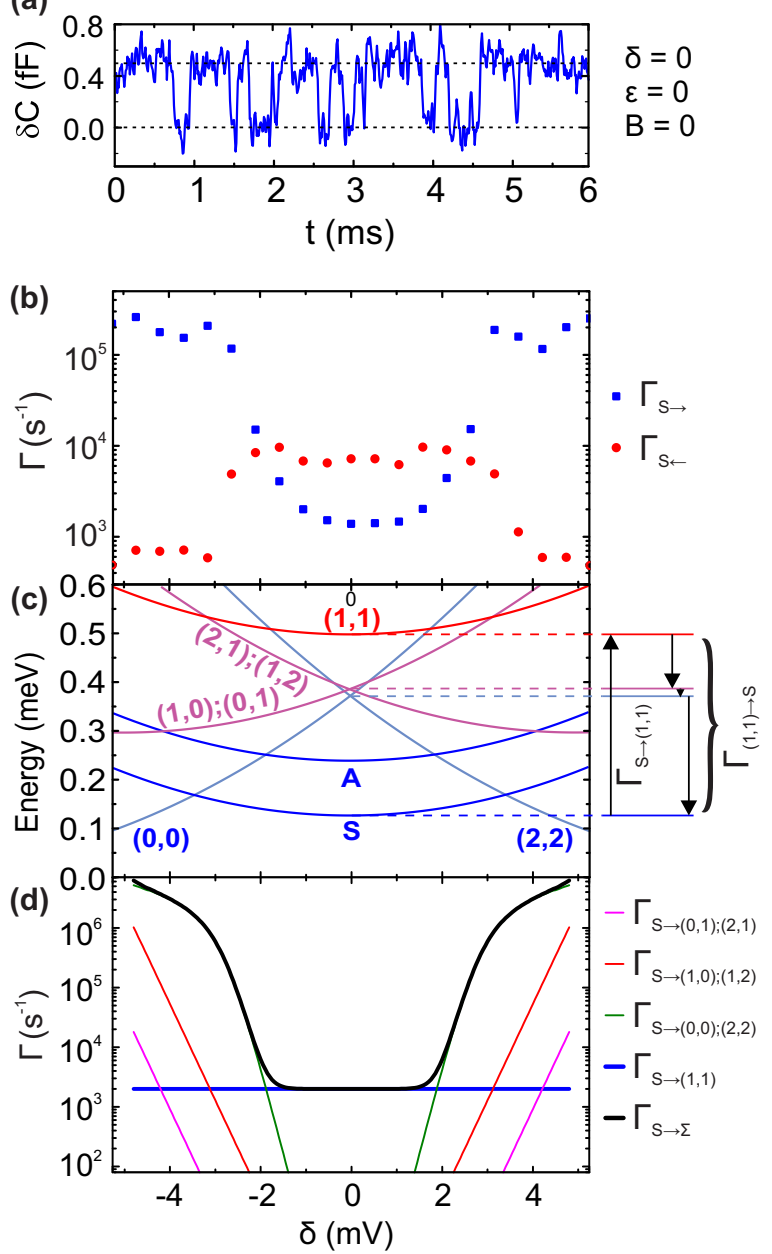

FIG. 3. (Color online) Splitting and relaxation. (a) Time domain measurement of $C_{Q}$ at $\epsilon=\delta=0$, showing transitions between $C_{Q}=0.5 \mathrm{fF}$ and $C_{Q}=0$ states. (b) Rates $\Gamma_{S \rightarrow}$ and $\Gamma_{S \leftarrow \text { determined }}$ from time domain measurements as a function of $\delta$ detuning. (c) Calculated band structure as a function of $\delta$. Dominant excitation and recombination routes at zero detuning are shown on the right. (d) Calculated rates for various transitions, using the model described in the text. $\Gamma_{S \rightarrow \Sigma}$ is the sum of all rates from the ground state. Around zero detuning, the $S \rightarrow(1,1)$ transition dominates.

Biasing at single points in gate voltage along the line $\epsilon=0$, and turning off the averaging in our data acquisition, we measure excursions of the system into excited states in the time domain [Fig. 3(a)]. This is characterized by the two-level switching between states with $C_{Q}=0.5 \mathrm{fF}$ and $C_{Q}=0$. While there is only one possible state with $C_{Q}=0.5 \mathrm{fF}$, there are a number of possible states with $C_{Q}=0$. These are the Cooper pair states $(0,0)$ and $(2,2)$, the odd-parity states $(0,1),(1,0)$, $(2,1),(1,2)$, and the doubly odd-parity state $(1,1)$ [Fig. 3(c)]. In order to determine which of the states are responsible for our measurement result, we analyze the switching data by collecting the switching times into a histogram. The result is fitted to the exponential distribution expected for a Poisson process, and then corrected for the finite bandwidth of our measurement system [29]. In this way we are able to find the rates from $\left(\Gamma_{S \rightarrow}\right)$ and to $\left(\Gamma_{S \leftarrow}\right)$ the symmetric ground state.
Plotting these rates as a function of $\delta$ detuning [Fig. 3(b)], we see that $\Gamma_{S \rightarrow}$ is flat in the region of $\delta=0$, whereas for larger values of $\delta$ it increases exponentially until our measurement is bandwidth limited. The rate of the $S \rightarrow(1,1)$ process is expected to be constant since it depends only on the energy difference between the $(1,1)$ state and $S$, which is independent of $\delta$. We therefore conclude that in the flat region of detuning, the $S \rightarrow(1,1)$ process is responsible for the switching. Our theoretical model corroborates this conclusion, as we calculate the other processes causing transitions from $S$ at $\delta=0$ to be orders of magnitude slower than the experimentally determined switching rate.

The exponential increase in rate with detuning can be understood by either the thermal activation of tunneling of single Cooper pairs or of single electrons, i.e., switching between $S$ and $(0,0)$ or $(2,2)$, or switching between $S$ and an odd-parity state such as $(1,0)$. To determine which is responsible, and to confirm the cause of the flat region, we examine theoretically the rates for all processes. Using existing formulas from earlier work on normal metal insulator superconductor (NIS) junctions, we calculate rates for quasiparticle tunneling [30] and Andreev reflection [31,32] from each of the leads. The former follow from Fermi's golden rule, while the latter are derived using a nonlinear response approach. Both of these rely on perturbative expansions of an electron tunneling Hamiltonian whose magnitude depends inversely on the junction resistance, which must therefore be suitably large for our theoretical results to be accurate; the relatively opaque lead junctions of our device justify this assumption. We choose a temperature of $125 \mathrm{mK}$ for these calculations, as we find $\Gamma_{S \rightarrow}$ to be constant below this point [Fig. 4(b)], suggesting the breakdown of thermalization. When the appropriate rates are summed, they agree qualitatively with the experimental data as long as a constant breaking rate is added. We attribute the constant rate to the $S \rightarrow(1,1)$ process, in which single Cooper pairs are being broken [Fig. 3(d)], as there are no other significant breaking processes with constant $\delta$ dependence. Furthermore, the simulations indicate that transitions from $S$ are primarily caused by Andreev reflection from the leads at larger $\delta$ detunings.

The recombination rate for a pair of quasiparticles in bulk aluminum corresponding to the SDD volume $(V \approx$ $\left.4 \times 10^{3} \mu \mathrm{m}^{3}\right)$ is $\frac{8(1.76)^{3}}{\tau_{0} V \rho_{0} \Delta} \approx 5 \mathrm{kHz}[33,34]$. This assumes an electron-phonon coupling constant $\tau_{0}=458 \mathrm{~ns}$ and a singlespin density of states for normal state aluminum $\rho_{0}=1.72 \times$ $10^{10} \mu \mathrm{m}^{-3} \mathrm{eV}^{-1}$. The equivalent direct recombination process for our SDD, $(1,1) \rightarrow S$, will be dramatically suppressed from the bulk rate due to the forced spatial separation of the quasiparticles. Therefore we expect recombination to occur via particle exchange with the leads. As $\delta$ detuning is increased, the ordering of the $(1,1)$ state and highest lying odd-parity state is reversed [Fig. 3(c)] and the recombination rate decreases, which we attribute to the requirement for thermal activation for the first step in these recombination paths. Note that the recombination cascade prevents the emission of a single phonon with energy $2 \Delta$, reducing any effect of the recombination phonon causing further pair-breaking events, as happens in bulk [35].

To further investigate the dynamics of the SDD we measure the rates $\Gamma_{S \rightarrow}$ and $\Gamma_{S \leftarrow \text { as a function of magnetic field }}$ [Fig. 4(a)] at $\epsilon=0, \delta=0$. We apply an in-plane magnetic 
(a) $\mathrm{T}=35 \mathrm{mK}$

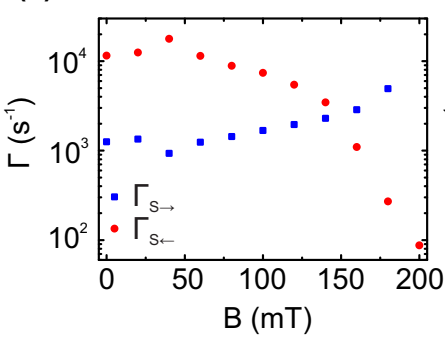

(b) $\mathrm{B}=0$

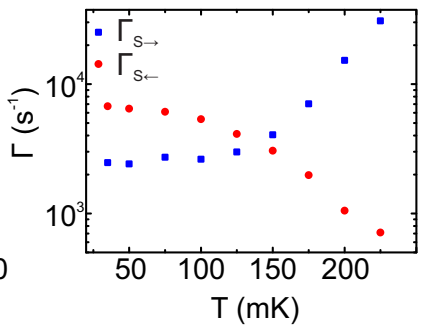

$\tilde{\Delta}(\mathrm{meV})$

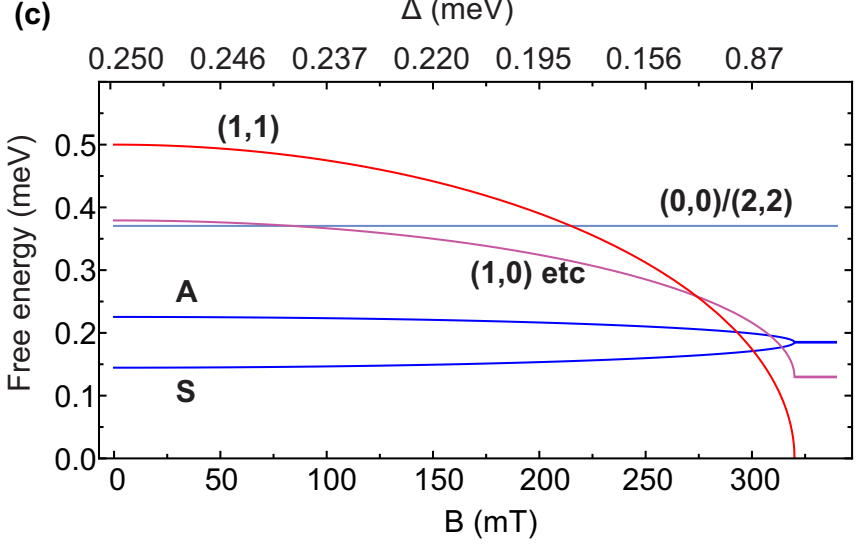

FIG. 4. (Color online) $\Gamma_{S \rightarrow}$ and $\Gamma_{S \leftarrow}$ as a function of (a) magnetic field at $T=35 \mathrm{mK}$, and (b) temperature at $B=0$. (c) Energy levels of device charge states at $\delta=\epsilon=0$ as a function of $B$, showing the change in state ordering. Equivalent values of $\tilde{\Delta}$ are shown on the top axis.

field which quenches superconductivity in the SDD at a critical field, $B_{c}=320 \mathrm{mT}$. The $B$ field suppresses the zero field gap $\Delta_{0}$ as $\Delta(B)=\Delta_{0} \sqrt{1-\frac{B^{2}}{B_{c}^{2}}}$ [36], where $B_{c}$ is the critical field, thus reducing the Josephson energy and hence the energy difference between $A$ and $S$ [Fig. 4(c)]. The quasiparticle containing states also have their energy suppressed as $\tilde{\Delta}$ is reduced, and the $(0,1)$ state is expected to be lower in energy than the $(0,0)$ state for magnetic fields above $\approx 75 \pm 25 \mathrm{mT}$. This leads to quasiparticle trapping in the $(1,0)$ state, which is released by thermally activated tunneling into $(0,0)$. $\Gamma_{S \leftarrow}$ therefore decreases with applied field past this point [Fig. 4(a)].

The rate $\Gamma_{S \rightarrow}$ increases with increasing field, since as $\tilde{\Delta}$ decreases the $(1,1)$ state lowers in energy and becomes more accessible. This observation is in line with previous measurements [14]. It is interesting to note that for $B>$ $150 \mathrm{mT}$, a population inversion occurs between $S$ and excited quasiparticle states as seen by the crossing of the $\Gamma_{S \rightarrow}$ and $\Gamma_{S \leftarrow \text { rates, and thus the trapped quasiparticle state dominates }}$ the population.

Finally, we monitor the rates as a function of temperature [Fig. 4(b)]. We find that $\Gamma_{S \rightarrow}$ has an approximately constant value at low temperature after which it increases with temperatures, and $\Gamma_{S \leftarrow \text { decreases with increasing temperature. This }}$ behavior can be understood in terms of the suppression of $\tilde{\Delta}$ by increasing temperature, which changes the ordering of the energy levels in a manner analogous to the case of field suppression.

In this work we have probed, at the single particle level, the rich quasiparticle dynamics of a SDD which allows single pair-breaking and recombination events to be observed. SDDs have future potential in the area of far-infrared light sensors since they provide the limiting case of a pair-breaking detector, in which a single far-infrared photon might create a single detected quasiparticle pair.

A.J.F. would like to acknowledge the Hitachi Research fellowship, support from Hitachi Cambridge Laboratory, and support from the EPSRC Grant No. EP/H016872/1. B.W.L. is supported by a Royal Society University Research Fellowship. F.A.P. would like to thank the Leverhulme Trust for financial support.
[1] M. T. Tuominen, J. M. Hergenrother, T. S. Tighe, and M. Tinkham, Phys. Rev. Lett. 69, 1997 (1992).

[2] P. Lafarge, P. Joyez, D. Esteve, C. Urbina, and M. H. Devoret, Phys. Rev. Lett. 70, 994 (1993).

[3] G. Catelani, S. E. Nigg, S. M. Girvin, R. J. Schoelkopf, and L. I. Glazman, Phys. Rev. B 86, 184514 (2012).

[4] P. de Visser, J. J. A. Baselmans, P. Diener, S. Yates, A. Endo, and T. M. Klapwijk, J. Low Temp. Phys. 167, 335 (2012).

[5] D. Rainis and D. Loss, Phys. Rev. B 85, 174533 (2012).

[6] P. K. Day, H. G. Leduc, B. A. Mazin, A. Vayonakis, and J. Zmuidzinas, Nature (London) 425, 817 (2003).

[7] R. Hanson, Rev. Mod. Phys. 79, 1217 (2007).

[8] P. Jarillo-Herrero, J. A. van Dam, and L. P. Kouwenhoven, Nature (London) 439, 953 (2006).

[9] L. Hofstetter, S. Csonka, J. Nygård, and C. Schönenberger, Nature (London) 461, 960 (2009).

[10] L. G. Herrmann, F. Portier, P. Roche, A. L. Yeyati, T. Kontos, and C. Strunk, Phys. Rev. Lett. 104, 026801 (2010).
[11] A. Das, Y. Ronen, M. Heiblum, D. Mahalu, A. V. Kretinin, and H. Shtrikman, Nat. Commun. 3, 1165 (2012).

[12] E. Bibow, P. Lafarge, and L. P. Lévy, Phys. Rev. Lett. 88, 017003 (2001).

[13] O. Naaman and J. Aumentado, Phys. Rev. B 73, 172504 (2006).

[14] A. J. Ferguson, N. A. Court, F. E. Hudson, and R. G. Clark, Phys. Rev. Lett. 97, 106603 (2006).

[15] R. M. Lutchyn, L. I. Glazman, and A. I. Larkin, Phys. Rev. B 74, 064515 (2006).

[16] D. Ristè, C. C. Bultink, M. J. Tiggelman, R. N. Schouten, K. W. Lehnert, and L. DiCarlo, Nat. Commun. 4, 1913 (2013).

[17] R. Barends, J. Wenner, M. Lenander, Y. Chen, R. C. Bialczak, J. Kelly, and E. Lucero, Appl. Phys. Lett. 99, 113507 (2011).

[18] K. J. Stone, K. G. Megerian, P. K. Day, P. M. Echternach, J. Bueno, and N. Llombart, Appl. Phys. Lett. 100, 263509 (2012).

[19] P. J. de Visser, J. J. A. Baselmans, S. J. C. Yates, P. Diener, A. Endo, and T. M. Klapwijk, Appl. Phys. Lett. 100, 162601 (2012). 
[20] N. J. Lambert, M. Edwards, C. Ciccarelli, and A. J. Ferguson, Nano Lett. 14, 1148 (2014).

[21] M. A. Sillanpää, T. Lehtinen, A. Paila, Y. Makhlin, L. Roschier, and P. J. Hakonen, Phys. Rev. Lett. 95, 206806 (2005).

[22] T. Duty, G. Johansson, K. Bladh, D. Gunnarsson, C. Wilson, and P. Delsing, Phys. Rev. Lett. 95, 206807 (2005).

[23] M. D. Shaw, J. Bueno, P. Day, C. M. Bradford, and P. M. Echternach, Phys. Rev. B 79, 144511 (2009).

[24] K. D. Petersson, C. G. Smith, D. Anderson, P. Atkinson, G. A. C. Jones, and D. A. Ritchie, Nano Lett. 10, 2789 (2010).

[25] W. G. van der Wiel, S. De Franceschi, J. M. Elzerman, T. Fujisawa, S. Tarucha, and L. P. Kouwenhoven, Rev. Mod. Phys. 75, 1 (2002).

[26] S. J. Chorley, J. Wabnig, Z. V. Penfold-Fitch, K. D. Petersson, J. Frake, C. G. Smith, and M. R. Buitelaar, Phys. Rev. Lett. 108, 036802 (2012).
[27] T. Frey, P. J. Leek, M. Beck, A. Blais, T. Ihn, K. Ensslin, and A. Wallraff, Phys. Rev. Lett. 108, 046807 (2012).

[28] V. F. Maisi, O.-P. Saira, Y. A. Pashkin, J. S. Tsai, D. V. Averin, and J. P. Pekola, Phys. Rev. Lett. 106, 217003 (2011).

[29] O. Naaman and J. Aumentado, Phys. Rev. Lett. 96, 100201 (2006).

[30] V. F. Maisi, S. V. Lotkhov, A. Kemppinen, A. Heimes, J. T. Muhonen, and J. P. Pekola, Phys. Rev. Lett. 111, 147001 (2013).

[31] F. W. J. Hekking, L. I. Glazman, K. A. Matveev, and R. I. Shekhter, Phys. Rev. Lett. 70, 4138 (1993).

[32] A. Hädicke and W. Krech, Phys. Status Solidi 191, 129 (1995).

[33] C. M. Wilson, L. Frunzio, and D. E. Prober, Phys. Rev. Lett. 87, 067004 (2001).

[34] P. J. de Visser, J. J. A. Baselmans, P. Diener, S. J. C. Yates, A. Endo, and T. M. Klapwijk, Phys. Rev. Lett. 106, 167004 (2011).

[35] A. Rothwarf and B. N. Taylor, Phys. Rev. Lett. 19, 27 (1967).

[36] M. Tinkham, Introduction to Superconductivity, 2nd ed. (Dover, Mineola, NY, 2004). 